\title{
ON MEASURABLE LOCAL HOMOMORPHISMS
}

\author{
ZOLTÁN SASVÁRI
}

(Communicated by Jonathan M. Rosenberg)

\begin{abstract}
We prove that every measurable local homomorphism between locally compact groups is continuous.
\end{abstract}

Let $G$ and $H$ be locally compact groups. In a recent paper A. Kleppner proved that every measurable homomorphism from $G$ into $H$ is continuous [2]. In the present note we show that this result is true for local homomorphisms as well. Our method of proof differs from that of [2]. The measure referred to throughout is a left Haar measure on $G$.

Theorem. Let $G$ and $H$ be locally compact groups and let $V$ be an open neighborhood of the identity of $G$. Suppose that $\varphi$ is a mapping from $V$ into $H$ such that

(i) $\varphi(x y)=\varphi(x) \varphi(y)$ whenever $x, y, x y \in V$;

(ii) $\varphi^{-1}(U)$ is measurable for every open set $U \subset H$.

Then $\varphi$ is continuous.

Proof. Let $U$ be an arbitrary neighborhood of the identity of $H$. To show $\varphi$ is continuous it is sufficient to show that $\varphi^{-1}(U)$ contains a neighborhood of the identity of $G$. Choose a symmetric, relatively compact open set $W \subset U, W \neq$ $\varnothing$ so that $W W \subset U$. If $\varphi^{-1}(W)$ is not locally null then by Corollary (20.17) in [1] the set $\varphi^{-1}(W) \varphi^{-1}(W)^{-1}$ contains a neighborhood of the identity. Since

$$
\varphi^{-1}(W) \varphi^{-1}(W)^{-1} \subset \varphi^{-1}(W W) \subset \varphi^{-1}(U),
$$

it remains to prove that $\varphi^{-1}(W)$ cannot be locally null.

On the other hand, suppose that $\varphi^{-1}(W)$ is locally null, and denote by $H_{0}$ the open subgroup generated by $W$. Choose a symmetric, relatively compact open set $V_{0} \subset V, V_{0} \neq \varnothing$ with $V_{0} V_{0} \subset V$. We show that $V_{0} \cap \varphi^{-1}\left(x H_{0}\right)$ is a null set for every $x \in H$.

Since $x H_{0}$ is $\sigma$-compact it can be covered by denumerably many sets of the form $y W(y \in H)$. Thus, it suffices to prove that $V_{0} \cap \varphi^{-1}(y W)$ is a null set for every $y \in H$. We put $E:=\varphi\left(V_{0}\right)$ and $S:=(E \cap y W)^{-}$. Because $S$ is compact

Received by the editors July 16, 1990.

1980 Mathematics Subject Classification (1985 Revision). Primary 22D05. 
and $S \subset \bigcup_{z \in E} z W$, there exists a finite number of elements $z_{1}, \ldots, z_{n} \in E$ such that

$$
S \subset \bigcup_{k=1}^{n} z_{k} W
$$

We have

$$
\begin{aligned}
& V_{0} \cap \varphi^{-1}(y W) \subset V_{0} \cap \varphi^{-1}(S) \subset V_{0} \cap\left(\bigcup_{k=1}^{n} \varphi^{-1}\left(z_{k} W\right)\right) \\
& =V_{0} \cap\left(\bigcup_{k=1}^{n} \varphi^{-1}\left(E \cap z_{k} W\right)\right) .
\end{aligned}
$$

Choose $g_{1}, \ldots, g_{n} \in V_{0}$ so that $\varphi\left(g_{k}\right)=z_{k}(k=1, \ldots, n)$. Using the relation $V_{0}^{-1} V_{0}=V_{0} V_{0} \subset V$ it is not difficult to see that

$$
V_{0} \cap \varphi^{-1}\left(E \cap z_{k} W\right)=V_{0} \cap g_{k} \varphi^{-1}(W) .
$$

It follows immediately from (1) and (2) that $V_{0} \cap \varphi^{-1}(y W)$ is a null set.

Now let $x_{\alpha}(\alpha \in \Gamma)$ be the set of left coset representatives of $H_{0}$. For an arbitrary index set $\Gamma_{0} \subset \Gamma$, the set $\bigcup_{\alpha \in \Gamma_{0}} x_{\alpha} H_{0}$ is open; so, $\bigcup_{\alpha \in \Gamma_{0}}\left(V_{0} \cap \varphi^{-1}\left(x_{\alpha} H_{0}\right)\right)$ is measurable. Moreover,

$$
V_{0}=\bigcup_{\alpha \in \Gamma}\left(V_{0} \cap \varphi^{-1}\left(x_{\alpha} H_{0}\right)\right),
$$

where the sets $V_{0} \cap \varphi^{-1}\left(x_{\alpha} H_{0}\right)$ are pairwise disjoint null sets. It follows from Theorem 3.1 in [3] that $V_{0}$ is a null set. This contradiction shows that $\varphi^{-1}(W)$ cannot be locally null.

\section{REFERENCES}

1. E. Hewitt and K. Ross, Abstract harmonic analysis. I, Springer-Verlag, New York, 1963.

2. A. Kleppner, Measurable homomorphisms of locally compact groups, Proc. Amer. Math. Soc. 106 (1989), 391-395.

3. J. Kupka and K. Prikry, The measurability of uncountable unions, Amer. Math. Monthly 91 (1984), 85-97.

Sektion Mathematik, Technische Universität, D-8027 Dresden, Germany 